\title{
A first-in-human oral dose study of mesdopetam (IRL790) to assess its safety, tolerability, and pharmacokinetics in healthy male volunteers
}

\author{
Folke Sjöberg ${ }^{1,2}$ @ | Susanna Waters ${ }^{3}$ | Boel Löfberg ${ }^{3}$ | Clas Sonesson ${ }^{3}$ | \\ Nicholas Waters $^{3}$ | Joakim Tedroff ${ }^{3,4}$
}

${ }^{1}$ Clinical Trials Consultants AB, Uppsala, Sweden

${ }^{2}$ Department of Biomedical and Clinical Sciences, Linköping University, Linköping, Sweden

${ }^{3}$ Integrative Research Laboratories AB, Gothenburg, Sweden

${ }^{4}$ Department of Clinical Neuroscience, Karolinska Institute, Stockholm, Sweden

\section{Correspondence}

Folke Sjöberg, Clinical trials Consultants AB, Dag Hammarskjölds väg 13, 75237

Uppsala, Sweden.

Email: folke.sjoberg@liu.se

\begin{abstract}
The management of Parkinson's disease (PD) is frequently compromised by complications induced by dopaminergic treatment such as involuntary movements (dyskinesias) and psychosis. Mesdopetam (IRL790) is a novel dopamine D3 receptor antagonist developed for the management of complications of therapy in PD. This study evaluated the safety, tolerability, and pharmacokinetics of escalating single and multiple doses of mesdopetam. We conducted a prospective, single-center, randomized, double-blind, placebo-controlled phase I, and first-in-human (FIH) study with mesdopetam administered to healthy male subjects. Overall, mesdopetam was welltolerated up to a $120 \mathrm{mg}$ single dose and up to $80 \mathrm{mg}$ upon multiple dosing. Adverse events (AEs) were mainly related to the nervous system and were dose-dependent. No serious adverse events occurred and no AEs led to withdrawal. The results of the single-ascending-dose and multiple-ascending-dose parts indicated dose- and timeindependent pharmacokinetics with rapid absorption and maximum plasma levels that were generally reached within $2 \mathrm{~h}$ after dosing. No accumulation was observed upon multiple dosing. It is concluded that mesdopetam was safe and well-tolerated in healthy male volunteers. Pharmacokinetic analysis indicated rapid absorption and dose-linear pharmacokinetics of mesdopetam, with a plasma half-life of around $7 \mathrm{~h}$, upon single and repeated dosing. The pharmacokinetics of mesdopetam supports twice-daily use in patients.
\end{abstract}

KEYWORDS

dopamine $D$ receptor, levodopa dyskinesia, Parkinson's disease, psychosis

Abbreviations: AE, adverse event; AR, accumulation ratio; AUC, area under the curve; CNS, central nervous system; C-SSRS, Columbia Severity Suicidal Rating Scale; ECG, electrocardiogram; FAS, full analysis set; FIH, first-in-human; GCP, Good Clinical Practice; GLP, Good Laboratory Practice; ICH, International Conference on Harmonization; LID, Levodopa (L-dopa)-Induced Dyskinesia; MAD, multiple-ascending-dose; NCA, non-compartmental analysis; PD, Parkinson's disease; PP, per-protocol; SAD, single-ascending-dose; TEAE, Treatment-Emergent AE.

The authors confirm that the Principal Investigator for this paper is Folke Sjoberg and that he had direct clinical responsibility for patients.

This is an open access article under the terms of the Creative Commons Attribution-NonCommercial-NoDerivs License, which permits use and distribution in any medium, provided the original work is properly cited, the use is non-commercial and no modifications or adaptations are made.

(c) 2021 The Authors. Pharmacology Research \& Perspectives published by British Pharmacological Society and American Society for Pharmacology and

Experimental Therapeutics and John Wiley \& Sons Ltd. 


\section{1 | INTRODUCTION}

Parkinson's disease (PD) is a relatively common neurodegenerative disorder characterized by motor symptoms such as poorness and slowness of movement, tremors, and loss of balance. Psychiatric symptoms such as anxiety and depression, as well as other nonmotor symptoms, are also common. ${ }^{1}$ Treatments that restore dopamine deficits in the brain such as levodopa and dopamine agonists have been used since the 1970s to treat the motor symptoms in PD but are known to cause adverse effects after a few years such as wearing-off, on-off, and dyskinesias. ${ }^{2}$ It is estimated that within 5 years of the initiation of standard dopamine replacement therapy in PD, about $50 \%$ of patients (and after 10 years almost all patients) develop involuntary movements, so-called Levodopa (L-dopa)Induced Dyskinesias (LIDs), in response to their medical treatment. ${ }^{3}$ LIDs are often the key complication limiting further dose increases in dopaminergic therapy.

Psychotic symptoms such as hallucinations and delusions are relatively common in patients with PD, when use of dopaminergic medication and cognitive impairment are the most important underlying factors. ${ }^{4}$ In a systematic review, the prevalence of hallucinations alone in patients with PD was between $21 \%$ and $46 \% .^{5}$

Mesdopetam (formerly IRL790, mesdopetam $\times 1 / 2$ L-tartrate $(\mathrm{N}$ [2-(3-fluoro-5-methylsulfonyl-phenoxy)ethyl]propan-1-amine)) is a novel dopamine D3 receptor antagonist. In experimental animal models, mesdopetam displays both antidyskinetic and antipsychotic properties, while leaving normal behavior largely unaffected, indicating a novel pharmacological profile with the potential to alleviate several troubling complications of therapy commonly seen in the management of PD. ${ }^{6}$

Mesdopetam is metabolized to two main pharmacologically inactive metabolites in man; the dealkylated M1 (IRL902) via CYP450, which is further acetylated by $\mathrm{N}$-acetyltransferase 2 (NAT2) to M2 (IRL872). Both metabolites are present in plasma and urine.

The objectives of this first-in-human (FIH) study were to assess the safety, tolerability, and pharmacokinetics of mesdopetam in healthy male volunteers after single and multiple oral dosing, including food effect on the pharmacokinetics after single dosing.

\section{2 | MATERIALS AND METHODS}

\section{1 | Study design}

This was a single-center, double-blind, randomized, placebocontrolled trial conducted between 23 November 2015 and 8 June 2016 at the CTC Clinical Trial Consultants Phase I unit in Uppsala, Sweden. Study code IRL790C001, EudraCT No 2015-003586-29.

The study comprised two parts.

Part 1 was designed as a partial cross-over single-ascending-dose (SAD) study with ascending dose levels of mesdopetam and one food interaction cohort. Sixteen (16) subjects were included in one of the two cohorts, eight subjects in each cohort (six active treatments and two placebos), and the cohorts were dosed in a zig-zag manner (5 mg-40 mg-160 mg and $15 \mathrm{mg}-80 \mathrm{mg}-\mathrm{food} / 80 \mathrm{mg}$ ) with a washout period of at least 1 week between doses. A follow-up visit was performed 5-10 days after the last dose.

Part 2 examined multiple-ascending-dose (MAD) cohorts with study treatment once daily for 10 consecutive days. Twenty-four (24) subjects were included, 12 subjects in each dose cohort (nine on active treatment, three on placebo), and the cohorts were dosed with 40 and $80 \mathrm{mg}$, respectively. A follow-up visit was performed 5-10 days after the last dose.

This study was approved by the Ethics Review Board in Uppsala, Sweden and conducted in compliance with the Declaration of Helsinki and the International Conference on Harmonization Guidelines for Good Clinical Practice. All subjects provided written informed written consent before participating in any study procedures.

\section{2 | Dose selection}

Based on preclinical results in experimental animal models, a clinically relevant plasma concentration of IRL790 is around $1 \mu \mathrm{M}$. The NOAEL in dogs given twice-daily oral doses IRL790 is $18 \mathrm{mg} / \mathrm{kg} /$ day (9 mg/kg orally twice daily), corresponding to $C_{\max }$ of about $9 \mu \mathrm{M}$. Calculations based on oral administration in dogs suggested that the starting dose in humans, $5 \mathrm{mg}$, would yield a peak plasma concentration of about $0.12 \mu \mathrm{M}$ and $1.3 \%$ of the plasma concentration of the NOAEL in the most sensitive species studied. The dose selection in the SAD part of the study was based on sub- and supra-clinical doses. Hence, the $40 \mathrm{mg}$ dose was calculated to yield peak plasma concentrations of about $1 \mu \mathrm{M}$ and the top dose, $160 \mathrm{mg}, 4 \mu \mathrm{M}$, well below the NOAEL level in dogs. Since dose-limiting AEs were expected to be central nervous system (CNS) related, it was our experience that humans could be more sensitive to such AEs, doses selected for the study did not a priori aim for an maximum tolerated dose level following single oral administration, but rather to cover a relevant plasma concentration range.

The partial cross-over design in the present study enabled each subject to receive maximum of three doses of IRL790; 5, 40, and $160 \mathrm{mg}$ or 15,80 (fasted), and $80 \mathrm{mg}$ (fed). The advantage of this two-cohort design was the ability to assess the dose-dependency of potential AEs in individual subjects but also to enable a sufficient number of subjects to evaluate the variability in pharmacokinetics (PK) properties.

Depending on the outcome of each dosing step, the investigational Safety Review Committee had the choice to decide to escalate the dose as planned, reduce, or increase the dose-escalation step, repeat the dose, reduce the dose, or terminate the study.

The MAD part of the study examined the effect of repeated once-daily dosing of a clinically relevant dose estimated to be in the range of 40-80 $\mathrm{mg}$ and a higher dose estimated to be in the range of $80-160 \mathrm{mg}$. The selection of the doses administered was determined depending on the results from the SAD part of the study. The maximum allowed exposure was to not exceed a $C_{\max }$ of $4.5 \mu \mathrm{M}$ and an $\mathrm{AUC}_{0-24}$ of $42 \mu \mathrm{M} / \mathrm{h}$. 
IRL790 is rapidly absorbed with a $t_{\max }$ of about $30-60$ min in experimental animals. Adverse effects are dose-dependent and CNSrelated, and appear to be most prominent around $C_{\max }$. The $60 \mathrm{~min}$ interval between dosing in the SAD part of the study would allow the investigator to adjust the schedule in case of intolerability in the higher dose groups. Moreover, since this was an FIH study, a 60 min interval between subjects would allow additional safety precautions upon initial dosing.

\section{3 | Subjects}

Healthy male subjects $18-50$ years of age, weighing at least $50 \mathrm{~kg}$, with a body mass index between 18 and $30 \mathrm{~kg} / \mathrm{m}^{2}$ were eligible for the study. Good health was determined by medical history, physical examination, vital signs, electrocardiogram (ECG), and laboratory tests at screening. Main exclusion criteria included: history of any clinically significant disease which, in the opinion of the investigator, could either put the subject at risk because of participation in the study or influence the results or the subject's ability to participate in the study.

\section{4 | Primary objectives and endpoints (safety and tolerability)}

In both the SAD and MAD part of the study, safety assessments included: frequency, seriousness, and intensity of adverse events (AEs), physical examination, 12-lead ECGs, Columbia Severity Suicidal Rating Scale, vital sign measurements, and laboratory measurements (clinical chemistry, hematology, and urinalysis). The SAD part also included telemetry.

\section{5 | Secondary objectives and endpoints (pharmacokinetic parameters)}

In the SAD part, blood samples for the measurement of plasma concentrations of mesdopetam and its metabolites IRL902 and IRL872 were collected at predetermined time points: predose, 20, $40 \mathrm{~min}, 1$ $2,3,4,6,8,10,12$, and $24 \mathrm{~h}$ after the intake of mesdopetam (up to $8 \mathrm{~h}$ only in the food interaction cohort). In the MAD part, blood samples were collected predose, 20, 40 min, 1, 2, 3, 4, 6, 8, 10, 12, and $24 \mathrm{~h}$ after the first and last dose of mesdopetam. A 48-h sample was also taken after the first dose, and on day 4 , sampling was performed predose, 1, 2, and $3 \mathrm{~h}$ postdose.

\section{6 | Bioanalytical methods}

Plasma samples were analyzed for mesdopetam and the metabolites IRL872 and IRL902 using ultra-performance liquid chromatographytandem mass spectrometry (UPLC-MS/MS) performed by the
National Veterinary Institute, Department of Chemistry, Environment and Feed Hygiene Section of Chemical Analysis, Uppsala, Sweden. A deuterated analog of each molecule was used as the internal standard. The method was validated according to EMA guidelines ${ }^{7}$ and the bioanalytical experiments performed in accordance with the OECD Principles of Good Laboratory Practice. The validated concentration range was $6.00-6000 \mathrm{nmol} / \mathrm{L}$ for mesdopetam, and 3.00-1000 and $1.00-1000 \mathrm{nmol} / \mathrm{L}$ for IRL872 and IRL902, respectively.

\section{7 | Statistical analysis}

No formal sample size calculation was performed for this study and therefore no hypothesis testing. The sample size was considered sufficient to provide adequate information for the study objectives.

The full analysis set, comprising all subjects who received randomized study treatment and have available data, has been used for the safety and tolerability assessments. Evaluations have been performed according to the actual treatment regardless of randomization.

The per-protocol (PP) analysis set comprises data from all randomized subjects who have received study treatment and have evaluable PK parameter data with no major protocol deviations with an impact on the PK data. All protocol violations were presented and discussed at the clean file meeting. The PP set has been used for the presentation of PK endpoints.

All statistical calculations were performed using the $\mathrm{SAS}^{\circledR}$ program (Version 90.4; SAS Institute Inc). The statistical analyses include descriptive statistics reflecting the explorative nature of the study.

\section{8 | Pharmacokinetic calculations}

The pharmacokinetic parameters were calculated by noncompartmental analysis (NCA) using the software Phoenix WinNonlin ${ }^{\circledR}$ version 6.3 or later (Pharsight Corporation). Plasma concentration values below the lower limit of quantitation and missing values (e.g., no blood sample collected, or no value obtained at analysis) were excluded from the NCA. Actual time-points for blood sampling were used in the calculations of the individual parameters, while nominal time points were denoted for summary statistics. Dose proportionality of $A U C$ and $C_{\max }$ was estimated using a non-linear power model. As an

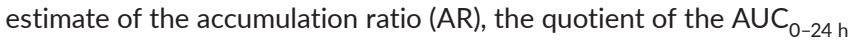
for the first and the last dose for each subject was calculated.

\section{3 | RESULTS}

\section{1 | Subject demographics}

A total of 40 subjects ( 17 subjects in part 1 and 23 subjects in part 2 ) were randomized to the study. All subjects were male Caucasians. One subject in part 1 discontinued after $5 \mathrm{mg}$ and was replaced 
for the subsequent dose levels, and one subject in part 2 withdrew consent before randomization and was not replaced. The treatment groups within each study part were comparable with respect to demographic parameters as well as concomitant medication and medical history.

\subsection{Safety and tolerability}

All 40 subjects were included in the safety analysis. There were no serious adverse events (SAEs) in the study and no AEs led to withdrawal.

The first subject administered a single dose at the highest predefined dose level, $160 \mathrm{mg}$, experienced adverse CNS symptoms of antidopaminergic character such as disturbance in attention, dizziness, tremor, restlessness, nervousness, and cold sweat. The main symptoms disappeared within $7 \mathrm{~h}$ postdose and the subject was fully recovered after 1 day. Due to these AEs, the dose was reduced to $120 \mathrm{mg}$ for the remaining subjects.

All AEs were coded according to MedDRA version 19.0. A detailed account of $A E s$ occurring after the first administration of investigational medical product (IMP) (Treatment-Emergent AEs, TEAEs) is presented by the SAD cohort (part 1) in Table S1 and by the MAD dose group (part 2) in Table S2. During single-dose escalation (part 1), the AEs most frequently represented were Infections and infestations (nasopharyngitis) and Nervous system disorders (headache being the most frequently reported event). Increased frequency or intensity of events by dose or by treatment (mesdopetam/placebo) was not seen in any of the AEs represented up to $120 \mathrm{mg}$ mesdopetam. At the $160 \mathrm{mg}$ dose level, the only subject treated experienced a disturbance in attention, dizziness, tremor, restlessness, nervousness, and cold sweat. Disturbance in attention and restlessness was also experienced by one (20.0\%) of the subjects given $120 \mathrm{mg}$ mesdopetam.

In part 2 (MAD) of the study, the AEs most frequently represented were Infections and infestations (nasopharyngitis), Nervous system disorders (somnolence, disturbance in attention, headache, and tremor), and Respiratory, thoracic, and mediastinal disorders (nasal congestion, epistaxis, and oropharyngeal pain). All 17 events reported within Nervous system disorders occurred after the administration of $80 \mathrm{mg}$ mesdopetam. Two subjects (25.0\%) reported a total of eight events of somnolence of which seven were assessed as probably related to the IMP and one as not related. One subject (12.5\%) reported seven events of disturbance of attention, and all were assessed as probably related to IMP.

Any TEAE was experienced at all dose levels in part 1 of the study, including placebo. Of the TEAEs reported, 20/35 (57.1\%) were assessed as not related to study treatment and $27 / 35$ (77.1\%) were of mild intensity. The one subject exposed to $160 \mathrm{mg}$ mesdopetam experienced AEs of moderate intensity and one mild event, all assessed as probably related to the IMP.

In part 2 of the study, any TEAE was experienced in all dose groups, including placebo. The proportion of subjects experiencing any TEAE at doses $40 \mathrm{mg}, 80 \mathrm{mg}$, and placebo was 55.6\%, 50.0\%, and $16.7 \%$, respectively. The average number of TEAEs reported by subject $(\mathrm{m} / \mathrm{n})$ was 1.2 and 5.8 at doses $40 \mathrm{mg}, 80 \mathrm{mg}$ and 1.0 for subjects given placebo. Following the administration of $80 \mathrm{mg}$ mesdopetam, 16/23 events reported (69.6\%) were assessed as possibly (2) or probably (14) related to the IMP whereas all events that occurred following the administration of $40 \mathrm{mg}$ mesdopetam or placebo were assessed as not related. Of the total 30 TEAEs reported, $66.7 \%$ were of mild intensity.

There were no remarkable mean changes over time or individual clinically significant abnormal findings with regards to any of the vital sign parameters and no abnormal findings upon physical examination were reported at any of the time-points assessed in the SAD or the MAD part of the study.

Electrocardiogram (Single 12-lead ECG in both parts 1 and 2 and ambulatory ECG telemetry in part 1 only) showed no remarkable mean changes over time or individual clinically significant abnormal values with regards to any of the ECG parameters measured.

Safety laboratory parameters showed no remarkable changes in mean values over time for any of the parameters analyzed.

Dopamine released from tuberoinfundibular dopamine neurons inhibits prolactin secretion from the anterior pituitary. ${ }^{8}$ As a biomarker for target engagement, plasma prolactin was measured at regular intervals in both part 1 and part 2 of the study. Following single dosing, mesdopetam produced a dose-dependent increase in plasma levels of prolactin indicating target engagement. Also, in the MAD part of the study modest, dose-dependent elevations of plasma prolactin were seen. Tables 1 and 2 shows the median plasma prolactin levels by dose and time after administration for the SAD and MAD parts of the study.

\section{3 | Pharmacokinetic assessments}

\subsection{1 | Single-dose pharmacokinetics}

All available mesdopetam concentration values for all subjects in all dose groups were included in the PK calculations. Following oral single-dose administration of mesdopetam, the plasma concentration increased rapidly to reach a maximum of 0.7 to $3 \mathrm{~h}$ after dosing, with a subsequent log-linear decline (Figure 1). The single-dose PK parameter results are presented in Table S3. In brief, for dose

TAB LE 1 Median plasma levels of prolactin $(\mu \mathrm{g} / \mathrm{L})$ at baseline (predose) and 2, 6, and $12 \mathrm{~h}$ and following single doses of mesdopetam

\begin{tabular}{llllr}
\multicolumn{1}{c}{ Baseline } & $2 \mathrm{~h}$ & $6 \mathrm{~h}$ & $12 \mathrm{~h}$ \\
Dose & & & & \\
$5 \mathrm{mg}$ & 15.0 & 20.5 & 8.3 & 10.0 \\
$15 \mathrm{mg}$ & 10.9 & 40.0 & 15.5 & 8.7 \\
$40 \mathrm{mg}$ & 12.5 & 54.0 & 23.0 & 13.5 \\
$80 \mathrm{mg}$ & 14.0 & 80.5 & 29.5 & 14.5 \\
$120 \mathrm{mg}$ & 12.5 & 68.0 & 29.5 & 19.5
\end{tabular}


TABLE 2 Median plasma levels of prolactin ( $\mu \mathrm{g} / \mathrm{L})$ at baseline (predose) and 2 and $12 \mathrm{~h}$ following multiple doses of mesdopetam

\begin{tabular}{|llll|}
\hline Dose/day & Baseline & $\mathbf{2 ~ h}$ & $\mathbf{1 2 ~ h}$ \\
\hline $40 \mathrm{mg} /$ day 1 & 13 & 39 & 14 \\
\hline $40 \mathrm{mg} /$ day 10 & 13 & 40 & 14 \\
\hline $80 \mathrm{mg} /$ day 1 & 14 & 61 & 15 \\
\hline $80 \mathrm{mg} /$ day 10 & 11 & 69 & 7 \\
\hline
\end{tabular}

levels 5-120 mg fasted, the following ranges were obtained for mesdopetam:

- Mean $t_{1 / 2}$ from $6.4 \pm 1.9 \mathrm{~h}$ to $7.1 \pm 1.0 \mathrm{~h}$ with the highest mean value after $80 \mathrm{mg}$ mesdopetam.

- Median $t_{\max }$ from $1.0 \mathrm{~h}$ (range 0.7-3.0) to $2.0 \mathrm{~h}$ (range 1.0-4.0) with the highest median value after $15 \mathrm{mg}$ mesdopetam.

- Mean $C_{\max }$ from $73.6 \pm 18.4 \mathrm{nmol} / \mathrm{L}$ to $1940 \pm 326 \mathrm{nmol} / \mathrm{L}$ with the highest mean value after $120 \mathrm{mg}$ mesdopetam.

- Mean $\mathrm{AUC}_{0-\infty}$ from $786 \pm 298 \mathrm{nmol} \mathrm{h} / \mathrm{L}$ to $17,300 \pm 4620 \mathrm{nmol}$ $\mathrm{h} / \mathrm{L}$ with the highest mean value after $120 \mathrm{mg}$ mesdopetam.

- Mean CL/F from $23.8 \pm 4.3 \mathrm{~L} / \mathrm{h}$ to $32.5 \pm 6.2 \mathrm{~L} / \mathrm{h}$ with the highest mean value after $80 \mathrm{mg}$ mesdopetam.

- Mean $V_{z} / F$ from $223 \pm 41.7 \mathrm{~L}$ to $328 \pm 59.0 \mathrm{~L}$ with the highest mean value after $80 \mathrm{mg}$ mesdopetam.

\subsection{Relative bioavailability after fed and fasting conditions}

For the four subjects receiving mesdopetam both under fasting and fed conditions, a small food interaction was observed. The geometric mean ratio (fed/fasted) was $110.7 \%$ for $\mathrm{AUC}_{0-8 \mathrm{~h}}$ and $109.0 \%$ for $C_{\max } \cdot t_{\max }$ was also delayed after food intake compared to fasting.

For the metabolites IRL902 and IRL872, the geometric mean ratio (fed/fasting) was $82.5 \%$ and $84.5 \%$ for $\mathrm{AUC}_{0-8 \mathrm{~h}}$ and $95.9 \%$ and $117.8 \%$ for $C_{\max }$ essentially reflecting the results for mesdopetam.

\subsection{Dose proportionality after single dose}

The analysis of dose linearity for $A \cup C_{t}, A \cup C_{0-\infty}$, and $C_{\max }$ showed a linear relationship with a proportionality constant (b) close to 1 , indicating dose proportionality for the parent compound mesdopetam and the metabolite IRL902.

\section{6 | Multiple-dose pharmacokinetics}

Mean plasma concentration-time profiles for mesdopetam after multiple dosing of 40 and $80 \mathrm{mg}$ once daily are shown

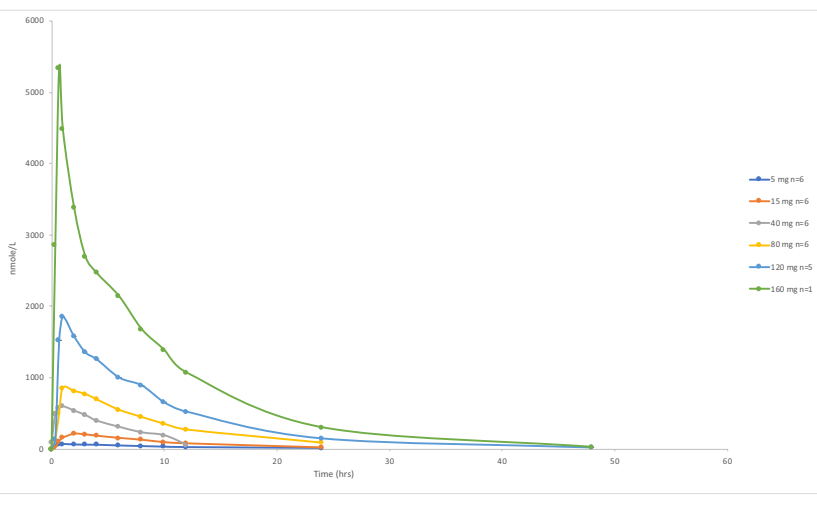

FIGURE 1 Plasma concentration-time curves following single oral doses of mesdopetam

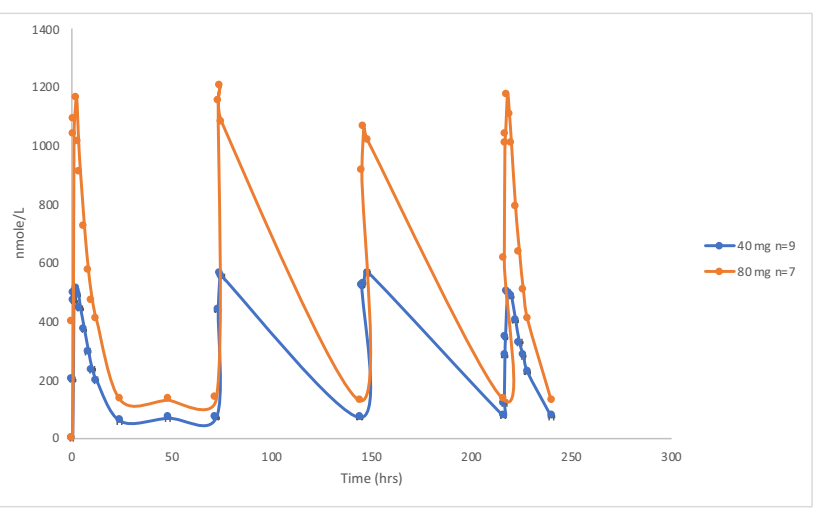

FIGURE 2 Plasma concentration-time curves following multiple oral doses of mesdopetam

in Figure 2. The individual concentration-time profiles for the first and last dose of $40 \mathrm{mg}$ mesdopetam show rapid absorption for the parent compound with median $t_{\max }$ at $2.0 \mathrm{~h}$ (range $0.3-3.0$ ) and $2.0 \mathrm{~h}$ (range $0.7-8.0$ ) and a mean $C_{\max }$ of $631 \pm 54.0 \mathrm{nmol} / \mathrm{L}$ and $567 \pm 95.7 \mathrm{nmol} / \mathrm{L}$ for the first and last dose, respectively. The $t_{1 / 2}$ was $6.8 \pm 1.3 \mathrm{~h}$ and $7.0 \pm 1.4 \mathrm{~h}$ for the first and last dose, respectively. Mean $\mathrm{AUC}_{0-24 \mathrm{~h}}$ after the first dose was $5580 \pm 1650 \mathrm{nmol} \cdot \mathrm{h} / \mathrm{L}$ and mean $A \cup C_{s s}$ was $6000 \pm 1970 \mathrm{nmol} \cdot \mathrm{h} / \mathrm{L}$ (Table S4).

After the first and last dose of $80 \mathrm{mg}$ mesdopetam, a mean $C_{\max }$ of $1490 \pm 410 \mathrm{nmol} / \mathrm{L}$ and $1430 \pm 393 \mathrm{nmol} / \mathrm{L}$ was reached at a median $t_{\max }$ of $0.8 \mathrm{~h}$ (range $0.3-2.0$ ) and $2.0 \mathrm{~h}$ (range 0.43.0), respectively. The $t_{1 / 2}$ was $7.1 \pm 1.1 \mathrm{~h}$ for the first dose and $7.2 \pm 0.6 \mathrm{~h}$ for the last dose. Mean $\mathrm{AUC}_{0-24 \mathrm{~h}}$ after the first dose was $11,500 \pm 2410 \mathrm{nmol} \cdot \mathrm{h} / \mathrm{L}$ and mean $A \cup C_{\text {ss }}$ was $12,200 \pm 3150 \mathrm{nmol} \cdot \mathrm{h} / \mathrm{L}$.

The time course of mesdopetam and IRL902 showed similar $t_{1 / 2}$ with lower concentrations for IRL902. IRL872 showed a longer $t_{1 / 2}$ as compared to the parent compound and IRL902; $46 \pm 43 \mathrm{~h}$ and $40 \pm 14 \mathrm{~h}$ after the first and last $40 \mathrm{mg}$ dose and $73 \pm 70 \mathrm{~h}$ after the last $80 \mathrm{mg}$ dose. The variations in IRL872 levels between subjects were approximately 10 -fold. 


\section{7 $\quad$ Dose proportionality after multiple doses}

The proportionality constant for $A \cup C_{s s}$ and $C_{\text {max }}$ of mesdopetam, for the last MAD dose, indicated dose proportionality for $\mathrm{AUC}_{\mathrm{ss}}(1.06)$ and supra-proportional increases of $C_{\max }$ (1.3).

\section{8 $\quad$ AR after multiple doses}

The mean AR for mesdopetam and IRL902 was close to 1 for both the 40 and $80 \mathrm{mg}$ dose level indicating virtually no accumulation from the first to the last dose which is in good agreement with the degree of accumulation calculated from the estimated half-lives and the dose interval. IRL872 showed accumulation at both dose levels, around twofold, in line with its longer half-life.

\section{4 | DISCUSSION}

Mesdopetam (formerly IRL790) is a novel compound for the treatment of dyskinesia and psychosis in PD. This was an FIH study investigating the safety, tolerability, and pharmacokinetics after single and repeated oral administrations of mesdopetam.

During the initial single-dose escalation, five escalating doses of IRL790 were tested in 16 male subjects included in two alternating cohorts with eight subjects in each. At each dose level, two subjects were given placebo and six subjects were given IRL790, using a partial cross-over design.

The multiple-dose escalation included 23 healthy male subjects. IRL790 was administered as once-daily doses for 10 consecutive days in doses 40 and $80 \mathrm{mg}$.

Overall, IRL790 was well-tolerated up to $120 \mathrm{mg}$ single dose and up to $80 \mathrm{mg}$ multiple doses although an increased frequency of AEs within Nervous system disorders (i.e., somnolence, disturbance in attention, headache, and tremor), assessed as probably related to study treatment, was seen after multiple dosing at the higher dose level (80 mg), as compared to $40 \mathrm{mg}$. No SAEs occurred and no AEs led to withdrawal.

The AEs that occurred in one subject administered a single dose of $160 \mathrm{mg}$ were assessed as being probably related to the study drug as antidopaminergic symptoms, and the dose was reduced to $120 \mathrm{mg}$ for the remaining subjects. These AEs were uncomfortable for the subject, but short-lasting and not serious.

There were no remarkable mean changes over time or individual clinically significant abnormal findings in vital signs, physical examination, or ECG parameters at any of the time-points assessed in the SAD or the MAD part of the study. Safety laboratory parameters did not show any significant abnormal changes. A modest, dose-dependent increase in s-prolactin was observed after both SAD and MAD parts of the study, consistent with target engagement at pituitary dopamine receptors.

\section{1 | Pharmacokinetics}

Following single doses of mesdopetam $A \cup C_{t}, A \cup C_{0-\infty}$ and $C_{\max }$ showed a dose-linear relationship indicating dose proportionality for mesdopetam. Urine recovery analysis showed that renal excretion amounted to around $30 \%$ of the dose administered, indicating renal excretion of the unchanged compound as one of the major pathways for the elimination of mesdopetam. The mean $t_{1 / 2}$ of the parent compound mesdopetam ranged from 6.4 to $7.1 \mathrm{~h}$. The plasma concentration-time profile of the metabolite IRL902 was similar to the profile for the parent compound which points to formation rate-limited pharmacokinetics, while the plasma concentration profiles for the metabolite IRL872 were markedly different with a mean $t_{1 / 2}$ of more than $20 \mathrm{~h}$ indicating elimination ratelimited pharmacokinetics for this metabolite. Since both IRL902 and IRL872 are pharmacologically inactive, no untoward pharmacological effects are expected from the metabolites formed from mesdopetam.

A small food interaction that suggested slightly higher exposures under fed conditions was observed for mesdopetam. After multipledose administration, the pharmacokinetic profile was similar to single-dose pharmacokinetics, with rapid absorption for the parent compound and a $t_{1 / 2}$ of $\sim 7 \mathrm{~h}$. The mean AR for mesdopetam was close to 1 for both 40 and $80 \mathrm{mg}$ dose levels indicating virtually no accumulation from the first to the last dose. One possible explanation for the supra-proportional increases of IRL902 levels between the two MAD doses could be capacity-limited metabolism to IRL872 (acetylation), which showed little change in exposure between the doses. The significance of this finding is, however, limited since only two dose levels were studied and was only observed in part 2 of the study. The overall pharmacokinetics of mesdopetam supports twice-daily use in patients. The dose of $40 \mathrm{mg}$ BID had fewer CNS $A E s$ but even a lower dose might be relevant for the patient with PD as they are more sensitive to CNS-related AEs. This is further supported by the conclusions made in a recent phase $1 \mathrm{~b}$ trial in patients with PD, where the average mesdopetam dose chosen was $18 \mathrm{mg}$ daily in the stable dose phase. ${ }^{9}$

In conclusion, mesdopetam was generally safe and welltolerated in healthy male subjects up to $120 \mathrm{mg}$ single dose and to $80 \mathrm{mg}$ in the MAD part. There were no notable safety findings or indications of a safety risk. Mesdopetam displayed rapid absorption and linear pharmacokinetics with an apparent terminal elimination half-life around $7 \mathrm{~h}$ upon single as well as repeated administration.

\section{ACKNOWLEDGMENTS}

The authors would like to acknowledge all investigators and staff involved at the study center. We also would like to direct our specific acknowledgments to Rikard Sandström for his excellent contributions to this manuscript. Unfortunately, he was unable to see the final result due to his unexpected and early death. 


\section{DISCLOSURE}

The following co-authors (SW, BL, CS, NW, and JT) have been supported by the sponsor (salary), Integrative Research Laboratories $A B$, Gothenburg, Sweden.

\section{AUTHOR CONTRIBUTION}

All the authors contributed to conception and design, collection and assembly of data, data analysis and interpretation, and manuscript writing, and provided final approval of the manuscript.

\section{DATA AVAILABILITY STATEMENT}

Data will be available from the first author on reasonable request.

\section{ORCID}

Folke Sjöberg (D) https://orcid.org/0000-0002-5903-2918

\section{REFERENCES}

1. Hely MA, Reid WG, Adena MA, Halliday GM, Morris JG. The Sydney multicenter study of Parkinson's disease: the inevitability of dementia at 20 years. Mov Disord. 2008;23:837-844.

2. Aquino CC, Fox SH. Clinical spectrum of levodopa-induced complications. Mov Disord. 2015;1:80-89.

3. Manson A, Stirpe P, Schrag A. Levodopa-induced-dyskinesias clinical features, incidence, risk factors, management and impact on quality of life. J Parkinson's Dis. 2012;2(3):189-198.

4. Zhu K, van Hilten JJ, Putter H, Marinus J. Risk factors for hallucinations in Parkinson's disease: results from a large prospective cohort study. Mov Disord. 2013;28(6):755-762.

5. Friedman JH. Parkinson's disease psychosis: update. Behav Neurol. 2013;27(4):469-477.
6. Waters S, Sonesson C, Svensson P, et al. Preclinical pharmacology of [2 -(3-fluoro-5-methanesulfonyl-phenoxy)ethyl](propyl)amine (IRL790), a novel dopamine transmission modulator for the treatment of motor and psychiatric complications in Parkinson disease. J Pharmacol Exp Ther. 2020;374(1):113-125.

7. Kollipara S, Bende G, Agarwal N, et al. International guidelines for bioanalytical method validation: a comparison and discussion on current scenario. Chromatographia. 2011;73:201-217. https://doi. org/10.1007/s10337-010-1869-2

8. Fitzgerald P, Dinan TG. Prolactin and dopamine: what is the connection? A review article. J Psychopharmacol. 2008;22(2 Suppl): 12-19.

9. Svenningsson P, Johansson A, Nyholm D, et al. Safety and tolerability of IRL790 in Parkinson's disease with levodopa-induced dyskinesia-a phase 1b trial. NPJ Parkinson's Dis. 2018;4(1). https://doi. org/10.1038/s41531-018-0071-3

\section{SUPPORTING INFORMATION}

Additional supporting information may be found online in the Supporting Information section.

How to cite this article: Sjöberg F, Waters S, Löfberg B, Sonesson C, Waters N, Tedroff J. A first-in-human oral dose study of mesdopetam (IRL790) to assess its safety, tolerability, and pharmacokinetics in healthy male volunteers. Pharmacol Res Perspect. 2021;9:e00792. https://doi. org/10.1002/prp2.792 